\title{
A LOWER BOUND FOR THE NUMBER OF DISTINCT EIGENVALUES OF SOME REAL SYMMETRIC MATRICES*
}

\author{
C.M. DA FONSECA ${ }^{\dagger}$
}

\begin{abstract}
This mostly expository note surveys and recovers a lower bound for the number of distinct eigenvalues of real symmetric matrices associated with a graph. The relation is established with the length of some paths of the underlying graph, using an improvement of an inequality involving the multiplicities of the eigenvalues. An interesting use of that number is observed. Some applications of the results to particular classes of graphs are considered.
\end{abstract}

Key words. Multiplicities, distinct eigenvalues, real symmetric matrices, graphs, paths, spectra.

AMS subject classifications. $05 \mathrm{C} 50$

1. Introduction. The study of the spectra of real symmetric (or Hermitian) matrices subordinated to a certain tree, and, in particular, of the number of distinct eigenvalues such acyclic matrices, has long been the subject of attention in diverse areas of applied, numerical, and theoretical linear algebra.

Largely motivated by the research by Parter [20], Genin and Maybee [9], and Wiener [22], on the location and multiplicity of eigenvalues of sign-symmetric matrices whose associated graphs are trees, the interest on this subject emerged again, one decade ago, mainly with the frequently cited papers $[14,15,18]$. On the other hand, many of the recent results were inspired by earlier seminal results of Godsil on matching polynomials [11, Chapter 1] and [12], sometimes overlooked by the linear algebra community, and surveyed by the author in [8].

Another important reference is the paper [19] by Nylen, where a recursive algorithm to compute minimum rank or, equivalently, the maximum multiplicity of a tree, is provided.

Recently, Kim and Shader introduced in [17] a new approach, based on the Smith Normal Form, to study the spectra of real symmetric matrices with a given graph. The authors gave an inequality between the diameter of a tree $T$ and the minimum number of distinct eigenvalues of all real symmetric matrices whose graphs are $T$,

\footnotetext{
* Received by the editors on June 10, 2009. Accepted for publication on July 31, 2010. Handling Editors: Roger A. Horn and Fuzhen Zhang.

${ }^{\dagger}$ Department of Mathematics, University of Coimbra, 3001-454 Coimbra, Portugal (cmf@mat.uc.pt). This work was supported by CMUC - Centro de Matemática da Universidade de Coimbra.
} 
recovering some known results. The same technique was also applied to a particular class of real symmetric unicycle matrices. These authors also classified the trees for which each of the associated acyclic matrices has distinct eigenvalues whenever the diagonal entries are distinct [16].

The thrust of this note, mainly of an expository nature, is to improve the bound obtained in [7] and, consequently, to recover a lower bound for the number of distinct eigenvalues of a real symmetric matrix in terms of the lengths of the paths not containing any edge of the cycles of the underlying graph. This bound is established in a very concise way and improves some known results. Several examples of families of graphs are considered.

2. The multiplicities of the eigenvalues of a graph. For a given (weighted) connected graph $G$, let $A=\left(a_{i j}\right)$ be the real matrix whose graph $G(A)$ is $G$. For more details on the notation used here, the reader is referred to $[7,8]$. We focus this work on the set

$$
\mathcal{S}(G)=\left\{A \in \mathbb{R}^{n \times n} \mid A \text { is symmetric and } G(A)=G\right\},
$$

i.e., the set of all $n$-by- $n$ real symmetric matrices sharing a common graph $G$ of order $n$. Nevertheless, all results can easily be extended to Hermitian matrices.

For a nontrivial (weighted) path $P$ in $G$, let us define $w(P)=\prod_{(i, j)} a_{i j}(P)$, where the product is taken over the weights of the edges $(i, j)$ of $P$, assuming that $i \neq j$. If the set of all paths connecting the vertex $i$ to the vertex $j$ is represented by $\mathcal{P}_{i j}$, the polynomial

$$
\varphi_{i j}(G, \lambda)=\sum_{P \in \mathcal{P}_{i j}} w(P) \varphi(G \backslash P, \lambda)
$$

can be regarded as the $i j$-entry of the classical adjoint of $\lambda I-A(G)$ [13, p. 1729], with the convention $\varphi_{i i}(G, \lambda)=\varphi(G \backslash i, \lambda)$. Observe that if there is one path connecting $i$ and $j$ containing no edges of any cycle of $G,(2.1)$ can be simplified.

Lemma 2.1. If there is only one path $P$ connecting two vertices $i$ and $j$ in a graph $G$, then $\varphi_{i j}(G, \lambda)=w(P) \varphi(G \backslash P, \lambda)$.

From (2.1) we can also deduce

$$
\varphi(G, \lambda) \varphi(G \backslash i, \mu)-\varphi(G, \mu) \varphi(G \backslash i, \lambda)=(\lambda-\mu) \sum_{j=1}^{n} \varphi_{i j}(G, \lambda) \varphi_{i j}(G, \mu),
$$

for any $i \in\{1, \ldots, n\}$. This identity is called the Christoffel-Darboux Identity for graphs by analogy with the celebrated identity for orthogonal polynomials [7, 12]. 
Several identities that can be derived from (2.2) are given in [7]. For example, we may state

$$
\varphi^{\prime}(G, \lambda)^{2}-\varphi^{\prime \prime}(G, \lambda) \varphi(G, \lambda)=\sum_{i, j=1}^{n} \varphi_{i j}(G, \lambda)^{2}
$$

Let us denote the (algebraic) multiplicity of the eigenvalue $\theta$ of a symmetric matrix $A=A(G)$ by $m_{A}(\theta)$. Using (2.3), motivated by the work on matching polynomials [12], we can give a lower bound for the multiplicity of $\theta$ as a zero of each $\varphi_{i j}(G, \lambda)$ :

TheOrem 2.2 ([7]). Let $P$ be a path in a graph $G$ and $A(G) \in \mathcal{S}(G)$. If $\theta$ is an eigenvalue of $A(G)$, then the multiplicity of $\theta$ as a zero of each $\varphi_{i j}(G, \lambda)$ is at least $m_{A(G)}(\theta)-1$.

Taking Lemma 2.1 into account, we obtain the following corollary.

Corollary 2.3. Let $P$ be a path that does not contain any edge of any cycle in G. Then

$$
m_{A(G \backslash P)}(\theta) \geq m_{A(G)}(\theta)-1 .
$$

EXAMPLE 2.4. We may try to extend (2.4) to paths containing a single edge of a cycle, if it exists, of the graph. Consider the matrix

$$
A=\left[\begin{array}{lllllll}
0 & 1 & 0 & 0 & 0 & 0 & 0 \\
1 & 0 & 1 & 0 & 0 & 0 & 0 \\
0 & 1 & 0 & 1 & 0 & 0 & 0 \\
0 & 0 & 1 & 1 & 1 & 0 & 1 \\
0 & 0 & 0 & 1 & 1 & 1 & 0 \\
0 & 0 & 0 & 0 & 1 & 1 & 1 \\
0 & 0 & 0 & 1 & 0 & 1 & 1
\end{array}\right],
$$

whose graph $G$ is the $(4,3)$-tadpole graph

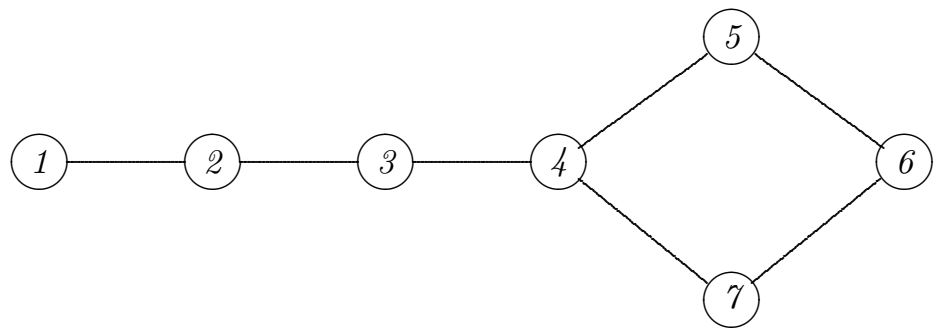

The eigenvalue 1 of $A$ has multiplicity 2 . If we remove the path $P$ joining the vertices 1 and 4, the multiplicity of 1 as eigenvalue of the submatrix $A(G \backslash P)$ is 1 ; but 
if we remove the shortest path joining the vertices 1 and 5, 1 is not an eigenvalue of the corresponding submatrix. Therefore, (2.4) cannot be extended to paths containing a single edge of a cycle.

Of course other examples can be given to show that (2.4) fails in general. For example, consider the 0-1 adjacency matrix of a complete graph and remove a single edge (including the terminal vertices). In Lemma 5.1 we identify the maximum multiplicity of an eigenvalue of a tadpole graph.

If one considers the trivial path reduced to a single vertex, say $i$, the inequality (2.4) becomes

$$
m_{A(G \backslash i)}(\theta) \geq m_{A(G)}(\theta)-1,
$$

which can also be seen as an immediate consequence of Cauchy's Interlacing Theorem for eigenvalues of symmetric matrices. It is a standard result that $M(G)-1 \leq$ $M(G-i) \leq M(G)+1$, where $M(G)$ denotes the maximum multiplicity of $G[19$, Proposition 2.1]. But, if instead of a vertex, we remove any path from a tree, (2.4) is a general result.

Corollary $2.5([7,8])$. Let $P$ be a path in a tree $T$ and let $A(T) \in \mathcal{S}(T)$. If $\theta$ is an eigenvalue of $A(T)$, then

$$
m_{A(T \backslash P)}(\theta) \geq m_{A(T)}(\theta)-1 \geq 0 .
$$

This corollary is an extension to general acyclic matrices of a result on matching polynomials [11, Theorem 4.5] or on 0-1 adjacency matrices of trees [10].

3. The number of distinct eigenvalues. The well-known relation between $q(T)$, the minimum of the number of distinct eigenvalues of a symmetric matrix whose graph is the tree $T$, and the number $d(T)$ of vertices in a longest path of $T$,

$$
q(T) \geq d(T)
$$

has been considered using various procedures $[7,12,17,18]$.

For some special families of trees the bound (3.1) can be improved, e.g., as in [17], where the Smith Normal Form is used to handle the case of a generalized $k$-whirl $(k \geq 2)$ on $n$ vertices. In this section, the inequality (3.1) is extended to graphs with at least one edge not contained in any cycle.

From Lemma 2.1 and Corollary 2.3, if $P$ is a path not containing edges of any cycle in $G$, then the inequality (2.4) ensures that

$$
\sum_{\theta}\left(m_{A(G)}(\theta)-1\right) \leq \sum_{\theta} m_{A(G \backslash P)}(\theta) \leq|V(G \backslash P)|,
$$


where the sums run over all distinct eigenvalues of $A(G)$. Therefore $q(T) \geq|V(P)|$.

TheOREM 3.1. If $P$ is a longest path not containing edges of any cycle in a connected graph $G$, then

$$
q(G) \geq|V(P)|
$$

We remark that, for $0-1$ adjacency matrices, Theorem 3.1 is general [5, p. 88]. We observe also that the proof of Theorem 3.1 is quite elegant, and the result implies $[18$, Theorem 4].

Since a tree has no cycles, we get immediately the following corollary.

Corollary $3.2([7,11,17,18])$. The inequality (3.1) is valid for any graph that is a tree.

The inequality (3.2) does not depend on the size of any cycle of the graph.

Kim and Shader in [17] provided a different lower bound on $q(G)$ for an infinite class of connected graphs $G$ [17, Figure 4].

THEOREM 3.3 ([17]). Let $G$ be a connected graph on $n$ vertices that consists of a connected subgraph $H$ on $m$ vertices containing vertices $v_{1}, v_{2}, v_{3}$ such that for all $r$, $s$, and $t$ with $\{r, s, t\}=\{1,2,3\}$, there exists a unique shortest path from $v_{s}$ to $v_{t}$ that does not contain $v_{r}$, and 6 legs $L_{1}, \ldots, L_{6}$ on $\ell$ vertices such that the end vertices of $L_{1}, L_{2}$ (resp. $L_{3}, L_{4}$ and $L_{5}, L_{6}$ ) are adjacent to vertex $v_{1}$ (resp. $v_{2}$ and $v_{3}$ ). Then

$$
q(G) \geq \frac{9 \ell}{4}-2 m+\frac{15}{2} .
$$

What happens in Theorem 3.3 if we omit the hypothesis of unicity of the shortest path from $v_{s}$ to $v_{t}$ that does not contain $v_{r}$, and the common size of the legs? If we assume instead that the longest path containing $L_{i}$ and $L_{i+1}$, for $i=1,3,5$, has $2 \ell+1$ vertices, then we get, from $(3.2)$,

$$
q(G) \geq 2 \ell+1 .
$$

Of course, under the conditions of Theorem 3.3, for small values of $m$, the bound (3.3) is better than (3.4). For example, for $m=3$ and $\ell=5$, inequality (3.3) provides $q(G) \geq 13$ and from (3.4) one gets $q(G) \geq 11$. But for a larger value of $m$, the same cannot be said. For example, for $m=6,(3.3)$ provides $q(G) \geq 7$. 
4. New frontiers for the number of distinct eigenvalues. Interestingly, research on the number of distinct eigenvalues of a matrix has moved far beyond matrix theory, to surprisingly unrelated new areas, such as quantum information science. For example, assuming that all entries of $A(G)$, the weights of $G$, are nonnegative, a continuous-time quantum random walk on $G$ is dictated by a time-varying unitary matrix using the Schrödinger equation with $A$ as the Hamiltonian [4]. The evolution of the quantum walk is given by the equation

$$
|\psi(t)\rangle=e^{-i t A}|\psi(0)\rangle
$$

where $|\psi(t)\rangle$ is the time-dependent amplitude vector on the vertices of $G$, and $|\psi(0)\rangle$ is the initial amplitude unit vector. The average probability of vertex $j$ is defined as

$$
\bar{p}_{j}=\lim _{T \rightarrow \infty} \frac{1}{T} \int_{0}^{T}|\langle j \mid \psi(t)\rangle|^{2} d t
$$

It was recently proved [4] that in a quantum walk on a weighted connected graph $G$ starting at an arbitrary vertex, say $j$, the average probability of the start vertex satisfies

$$
\bar{p}_{j} \geq \frac{1}{\tau(G)},
$$

where $\tau(G)$ denotes the number of distinct eigenvalues of the adjacency matrix $A(G)$.

The notion of quantum random walks, one of the many surprising aspects of quantum information, was introduced in 1992, by Aharonov, Davidovich, and Zagury in [1], as the counterpart of classical random walks for particles that cannot be precisely localized due to quantum uncertainties.

5. Back to the multiplicities of eigenvalues. One of the consequences of Corollary 2.3 is the eminent result stating that all eigenvalues of any path or, equivalently, of any (irreducible) tridiagonal matrix, are simple, since $q(P)=d(P)=|V(P)|$. The same fact can be derived from Corollary 2.5, or from the fact that all the eigenvalues of an unreduced upper Hessenberg matrix are simple. Therefore, the multiplicity of any eigenvalue of the cycle on $n$ vertices, $C_{n}$, is at most $2[6,19,21]$. In fact, $C_{n} \backslash i$ is a path, for any vertex $i$, and for any eigenvalue $\theta$ of $A\left(C_{n}\right)$, from (2.5), we get

$$
1 \geq m_{A\left(C_{n} \backslash i\right)}(\theta) \geq m_{A\left(C_{n}\right)}(\theta)-1 \geq 0
$$

The same argument can be used for other classes of graphs, such as the tadpole graphs or any generalized kite graph, as in the following example: 


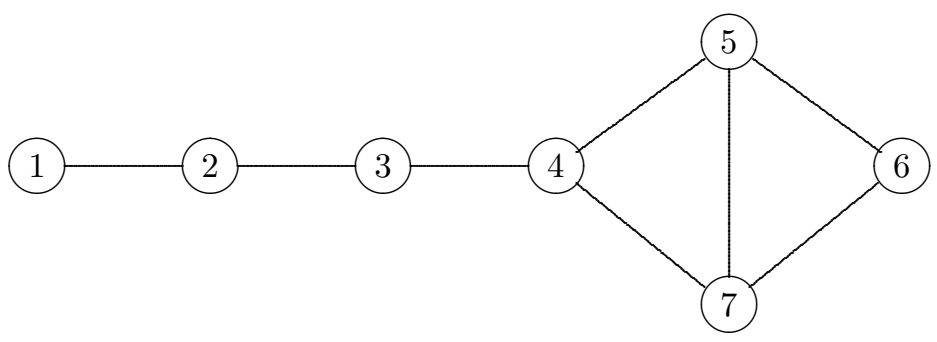

If one considers the vertex 5 (or 7 ) we get the following:

Lemma 5.1. The maximum possible multiplicity of an eigenvalue of a tadpole graph or a generalized kite graph is 2 .

But, for other classes of similar graphs, the inequality (5.1) does not provide the best bound. For example, let us consider the following generalized dart graph, say $D$ :

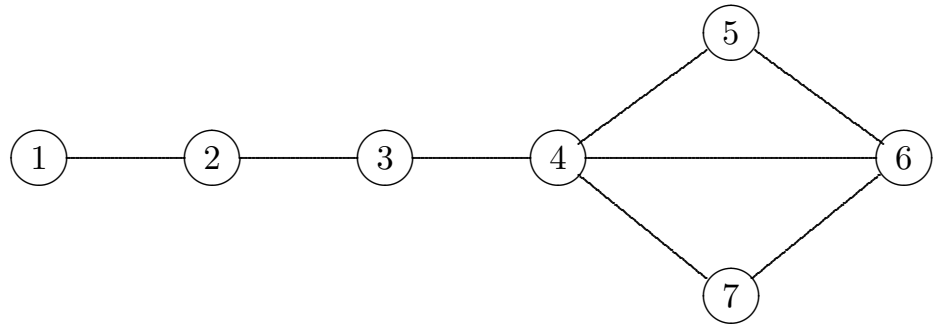

In this case, Theorem 3.1 guarantees that for the path $P$ joining vertices 1 and 4 , since $D \backslash P$ is a path,

$$
1 \geq m_{A(D \backslash P)}(\theta) \geq m_{A(D)}(\theta)-1 \geq 0 .
$$

A similar strategy can be applied to the parapluie graph [3]:

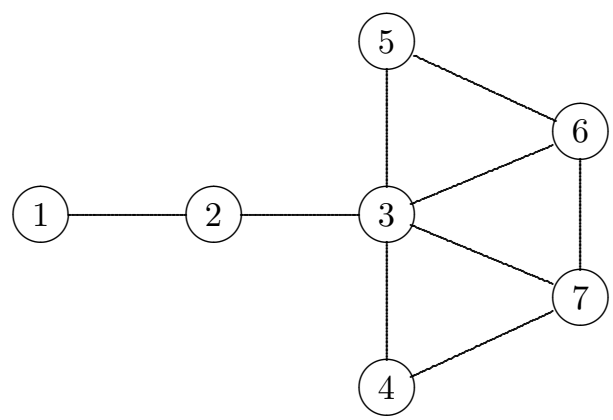


LEMma 5.2. The maximum possible multiplicity of an eigenvalue of a generalized dart graph or of the parapluie graph is 2 .

We conclude this note with a new application of Corollary 2.3. In 2004, Barioli et al. [2] determined the maximum possible multiplicity of an eigenvalue of the $n$-sun graph, i.e., the graph on $2 n$ vertices obtained by appending a leaf on each vertex of an $n$-cycle. ${ }^{1}$ Recently Sinkovic [21] discussed analogous properties of a larger family of graphs.

For the case of a 3-sunlet graph, also known as a net graph, Barioli et al. proved that the maximum eigenvalue multiplicity of such graphs is 2 . If one deletes one of the appending edges (including the terminal vertices), the resulting graph is a path. From (2.4), we conclude that the maximum eigenvalue multiplicity of a 3 -sunlet graph is at most 2 . This maximum is attained by the $0-1$ adjacency matrix of the 3 -sunlet graph.

The same procedure can be used to prove that 2 is the maximum eigenvalue multiplicity of a generalized 3-sunlet graph, i.e., the graph on $k n$ vertices obtained by appending a path on $k$ vertices on each vertex of an $n$-cycle.

Acknowledgment. The author thanks the referee for helpful comments concerning the paper.

\section{REFERENCES}

[1] Y. Aharonov, L. Davidovich, and N. Zagury. Quantum random walks. Phys. Rev. A, 48:16871690, 1993.

[2] F. Barioli, S. Fallat, and L. Hogben. Computation of minimal rank and path cover number for certain graphs. Linear Algebra Appl. 392:289-303, 2004.

[3] A. Brandstädt, V.B. Le, and J.P. Spinrad. Graph Classes: A Survey. SIAM Monographs on Discrete Mathematics and Applications, Philadelphia, PA, 1999.

[4] W. Carlson, A. Ford, E. Harris, J. Rosen, C. Tamon, and K. Wrobel. Universal mixing of quantum walk on graphs. Quantum Inf. Comput., 7:738-751, 2007.

[5] D.M. Cvetković, M. Doob, and H. Sachs, Spectra of graphs. Theory and application. Pure and Applied Mathematics, 87. Academic Press, Inc., New York-London, 1980.

[6] R. Fernandes and C.M. da Fonseca. The inverse eigenvalue problem for Hermitian matrices whose graphs are cycles. Linear Multilinear Algebra, 57:673-682, 2009.

[7] C.M. da Fonseca. A note on the multiplicities of the eigenvalues of a graph. Linear Multilinear Algebra, 53:303-307, 2005.

[8] C.M. da Fonseca. On the multiplicities of eigenvalues of a Hermitian matrix whose graph is a tree. Ann. Mat. Pura Appl. 187:251-261, 2008.

[9] J. Genin and J.S. Maybee. Mechanical vibration trees. J. Math. Anal. Appl., 45:746-763, 1974.

[10] C.D. Godsil. Spectra of trees, Ann. Discrete Math., 20:151-159, 1984.

\footnotetext{
${ }^{1}$ We remark that in the literature the term $n$-sun is reserved for a quite different class of graphs [3]. The term $n$-sunlet graph seems more appropriate.
} 
[11] C.D. Godsil. Algebraic Combinatorics. Chapman and Hall, New York and London, 1993.

[12] C.D. Godsil. Algebraic matching theory. Electron. J. Combin. 2: Research Paper 8, 1995.

[13] C.D. Godsil. Tools from linear algebra. Handbook of Combinatorics (eds., Graham, Grötschel, Lovász), Elsevier and MIT Press, 1995, 1705-1748.

[14] C.R. Johnson and A. Leal Duarte. The maximum multiplicity of an eigenvalue in a matrix whose graph is a tree. Linear Multilinear Algebra, 46:139-144, 1999.

[15] C.R. Johnson and A. Leal Duarte. On the possible multiplicities of the eigenvalues of an Hermitian matrix whose graph is a given tree. Linear Algebra Appl., 348:7-21, 2002.

[16] I-J. Kim and B.L. Shader. Classification of trees each of whose associated acyclic matrices with distinct diagonal entries has distinct eigenvalues. Bull. Korean Math. Soc., 45:95-99, 2008.

[17] I-J. Kim and B.L. Shader. Smith normal form and acyclic matrices. J. Algebraic Combin. 29:63-80, 2009.

[18] A. Leal-Duarte and C.R. Johnson. On the minimum number of distinct eigenvalues for a symmetric matrix whose graph is a given tree. Math. Inequal. Appl. 5:175-180, 2002.

[19] P.M. Nylen. Minimum-rank matrices with prescribed graph. Linear Algebra Appl., 248:303-316, 1996.

[20] S. Parter. On the eigenvalues and eigenvectors of a class matrices. J. Soc. Indust. Appl. Math., 8:376-388, 1960 .

[21] J. Sinkovic. Maximum nullity of outerplanar graphs and the path cover number. Linear Algebra Appl., 432:2052-2060, 2010.

[22] G. Wiener. Spectral multiplicity and splitting results for a class of qualitative matrices, Linear Algebra Appl., 61:15-29, 1984. 